be maintained that the water had been lost after the giant halo had been produced. It might also be thought that there should be some evidence of the halo produced by the alpha particles themselves, but as shown in the figure this would be removed by the annealing process at high levels of ionisation.

\section{Muonium hyperfine structure}

\section{from Peter Knight}

THE muon is a curious particle. It behaves in all respects like a heavy electron, yet there seems to be no explanation of the large ratio of the muon mass to the electron mass. The atom muonium, consisting of a positive muon and an electron, is the simplest system available for precise investigation of the interactions between these so nearly identical particles. Everything we currently know about muonium is completely specified by Quantum Electrodynamics (QED) and the Bethe-Salpeter relativistic twobody equation. Its hyperfine structure is similar to that of hydrogen, but avoids problems of hadron structure so a precision measurement of the muonic hyperfine structure (hfs) checks leptonic QED to high accuracy and could perhaps indicate differences between muons and electrons. V. W. Hughes of Yale University has undertaken a systematic programme of precision experiments on muonium for the past 20 years, and with 11 co-authors has recently reported some greatly improved results on the ground state hfs (Casperson et al. Phys. Rev. Lett. 38, 956; 1977).

Past work on the comparison of experiment with theory for muonium hfs has been limited by uncertainties in fundamental constants such as the fine structure constant $\alpha$ (this was most important), the velocity of light $c$ and the Rydberg constant $R_{\infty}$. These constants are now known to higher accuracy. Experimental work was limited by the number of muonium atoms available. The basic principles underlying the latest experiment have remained more or less unchanged from earlier work, and the improvement in accuracy has been made possible by using the higher muon intensity produced by the Los Alamos 'meson factory' LAMPF. Muons are produced by the parity-violating pion decay $\pi^{+} \rightarrow \mu^{+}+v_{\mu}$ and are spinpolarised with the muon spin direction opposed to the linear momentum. Parity violation not only provides polarised

Peter Knight is Jubilee Research Fellow in the Department of Physics at Royal Holloway College, University of London. muons but also provides an elegant monitor of the subsequent evolution of the muon spin. The positive muon decays into a positron and two neutrinos with the positron emitted preferentially along the muon spin direction (and therefore in an opposite direction to the muon momentum). Atomic muonium is formed by charge capture from a krypton gas target at 1.7 and 5.3 atmospheres. The charge capture process does not alter the muonic spin directions so that spin polarised muonium is formed.

In zero magnetic field there are two hyperfine structure levels-a triplet state with total angular momentum $F=1$ and a singlet state with $F=0$ separated from the triplet by the hyperfine structure interval $\Delta v$, caused by the magnetic interaction between the spin magnetic moments of the electron and the muon. In the strong magnetic field (13.6 kG) used by Casperson $e t$ al. the interaction of the magnetic moments with the external field is large compared with $\Delta v$. Figure 1 illustrates the magnetic-field dependence and state-labelling of the relevant $1^{2} \mathrm{~S}_{t}$ levels (the Breit-Rabi diagram). The relevant good quantum numbers are $M_{\mathrm{J}}$, the magnetic quantum number for the electron, and $M_{\mu}$ the muon spin magnetic quantum number.

The external magnetic field is in the opposite direction to the muon spin, so $M_{\mu}=-\frac{1}{2}$ and the states $\left(\frac{1}{2},-\frac{1}{2}\right)$ and $\left(-\frac{1}{2},-\frac{1}{2}\right)$ labelled respectively 2 and 3 are created in equal proportions. If these muonium atoms are left to decay, the decay positrons are emitted preferentially 'backwards'-opposed both to the muon beam direction and the external field direction. Resonant microwave fields induce transitions at frequencies $v_{12}$ and $v_{34}$ between states $2 \rightarrow 1$ and $3 \rightarrow 4$; the muon spin direction changes and the decay positrons are then emitted preferentially in the 'forward' direction of the external field. In other words, resonant transitions are detected by the change in the angular distribution of the decay positrons. Sets of proportional counters and scintillation counters are placed on both sides of the target in a $13.6 \mathrm{kG}$ external magnetic field produced by a precision solenoid.

Casperson et al. have measured $v_{12}$ and $v_{34}$. Measurement of both eliminates the need for a separate NMR measurement of the muon magnetic moment with its associated uncertainties. The combination $\left(v_{12}+v_{34}\right)$ is used to determine $\Delta v$ and $\left(v_{34}-v_{12}\right)$ to determine the muon magnetic moment $\mu_{\mu}$ in terms of the proton magnetic moment $\mu_{\mathrm{p}}$. Their results are $\Delta v=4463302.35$ (52) $\mathrm{kHz}$ (three or four times more precise than earlier measurements) and $\left(\mu_{\mu} / \mu_{p}\right)=$ 3.1833403 (44). This latter can be used to obtain a new, most precise value for the muon-electron mass ratio $\left(m_{\mathrm{u}} / m_{\mathrm{e}}\right)=$ 206.76859 (29). The difference between the theoretical value for $\Delta v$ (Brodsky \&

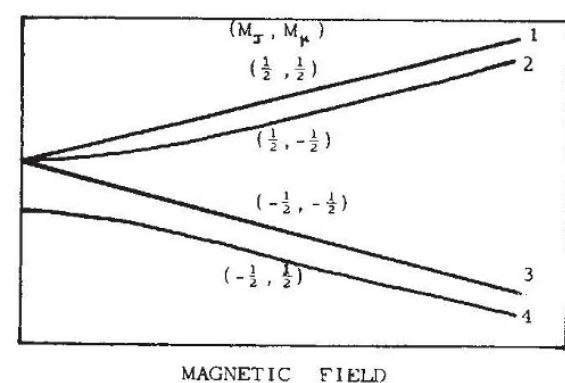

Fig. 1 Schematic diagram of Zeemun splitting of muonium hfs levels. At zero field, energy separation is $\Delta v$, the hfs interval.

Drell A. Rev. nucl. Sci. 20, 147; 1970) and the new experimental value is $16.1(6.6) \mathrm{kHz}, 2.5$ standard deviations of the combined error. The theoretical value is made up of several terms and the discrepancy may be due to an incomplete knowledge of the higher order radiative and recoil corrections to the bound state energy. These calculations are extremely complicated (for example Fulton \& Repko Phys. Rev. D14, 1243; 1976). Weak interaction contributions such as neutral current effects are discounted by Casperson et al. On the other hand, the measured density shifts of $v_{12}$ and $v_{34}$ indicate room for further experimental work.

\section{Biological sun-traps}

\section{from J. Coombs}

A European Seminar on Biological Solar Energy Conversion Systems was held in Grenoble-Autrans on 9-12 May, 1977. It was organised by Professor D. O. Hall, Kings College, University of London and Dr P. M. Vignais, CNRS, Grenoble, and sponsored by a number of French research agencies.

THE idea of solar energy has risen like the phoenix from the ashes of the world's fossil fuel reserves. The number of publications, reports and research proposals grows steadily, but how realistic are they? Some 300 scientists assembled at Autrans, under overcast skies and persistent drizzle to review the present state of the application of biological solar energy conversion.

The basic tenets remained unquestioned-that solar energy represents the only significant safe, renewable, non-polluting future source

J. Coombs is responsible for agricultural and biological research at the Philip Lyle Research Laboratory (Group Research and Development) Tate and Lyle Ltd, at the University of Reading. 